\title{
Quality of life in patients with type 2 diabetes after switching to insulin degludec: results from a cross-sectional survey
}

\author{
Chioma Uzoigwe ${ }^{1}$ (D) Michael Radin ${ }^{1} \cdot$ Carol M. Hamersky ${ }^{1} \cdot$ Mitch DeKoven $^{2} \cdot$ Cassie Holt $^{3} \cdot$ Swapna Karkare $^{4}$. \\ William H. Polonsky 5,6
}

Accepted: 28 December 2020 / Published online: 7 February 2021

(c) The Author(s) 2021

\begin{abstract}
Purpose Five quality of life (QoL) domains are particularly important to patients with type 2 diabetes (T2D) using basal insulin_-sense of physical well-being, sense of safety regarding hypoglycemia, sense of diabetes as burdensome, feelings of freedom and flexibility, and sleep quality.

Methods An online survey assessed these QoL domains in adult patients with T2D in the USA who had switched from a previous basal insulin to insulin degludec (IDeg): modified versions of the World Health Organization (Five) Well-Being Index (WHO-5), Hypoglycemia Attitudes and Behavior Scale (HABS; confidence and anxiety subscales only), and Diabetes Distress Scale (DDS; emotional burden and regimen-related distress subscales only); three items assessing feelings of freedom and flexibility; and one item assessing sleep quality (hours of restful sleep). Patients rated each item for their previous basal insulin and currently while using IDeg. Correlations between sleep quality and the other QoL scales were also assessed. Results In total, 152 patients completed the survey and were included in the study sample. Patients reported significantly improved scores while using IDeg on all WHO-5, DDS, HABS, feelings of freedom and flexibility item scores, and total raw/mean subscale scores $(P<0.0001)$. Patients also reported a significantly greater number of hours of restful sleep [mean $(S D) 6.6$ (2.0) vs. 5.5 (1.8); $P<0.0001]$. Better sleep quality statistically significantly correlated with improved QoL in all other domains assessed.

Conclusions Treatment with IDeg after switching from a previous basal insulin was associated with statistically significant improvements in all QoL domains assessed.
\end{abstract}

Keywords Quality of life · Type 2 diabetes $\cdot$ Basal insulin $\cdot$ Insulin degludec $\cdot$ Insulin glargine $\cdot$ Hypoglycemia

Supplementary Information The online version contains supplementary material available at https://doi.org/10.1007/s1113 6-020-02753-6.

\section{Chioma Uzoigwe}

coms@novonordisk.com

1 Novo Nordisk Inc., 800 Scudders Mill Road, Plainsboro, NJ 08536, USA

2 IQVIA, Falls Church, VA, USA

3 IQVIA, New York, NY, USA

4 IQVIA, Deerfield, IL, USA

5 Behavioral Diabetes Institute, San Diego, CA, USA

6 University of California, San Diego, CA, USA

\section{Introduction}

The addition of basal insulin is a well-established approach in patients with type 2 diabetes (T2D) who require intensification of antihyperglycemic therapy [1, 2]. Insulin degludec (IDeg) is one of several second-generation long-acting insulin analogs approved in the USA to improve glycemic control in patients with diabetes mellitus, available in the USA as of January 2016 [3, 4]. IDeg has a long duration of action and offers the convenience of once-daily dosing at any time of day $[3,5]$. Studies of the blood glucose-lowering effect of IDeg have shown that IDeg has a flatter, more stable pharmacodynamic profile compared with insulin glargine U-100 and U-300 [5, 6]. In addition, randomized controlled trials have found that IDeg is associated with significantly lower rates of severe hypoglycemia and nocturnal hypoglycemia than is insulin glargine U-100 in patients with T2D while 
providing equivalent glycemic control [7-9]. However, success of antihyperglycemic treatments in real-world clinical practice is determined not only by their clinical effectiveness and safety profile, but also by a range of additional factors that may affect treatment satisfaction and, hence, adherence [10]. Therefore, to obtain a comprehensive understanding of the potential value of a diabetes intervention, it is important to evaluate treatment outcomes in the broader context of treatment-related factors that may impact quality of life (QoL) [10].

The impact of IDeg on QoL in patients with T2D was previously assessed in a meta-analysis of three clinical trials of 26- or 52-weeks' duration in which patients were randomized to receive IDeg or insulin glargine U-100 [11]. Compared with insulin glargine U-100, IDeg improved both mental and physical health status on the widely used generic 36-item Short Form (SF-36) questionnaire. Anecdotal evidence has suggested that treatment with IDeg may be associated with unique and potentially important QoL benefits, which are often reported by patients simply as "feeling better" [12]. These findings are supported by a qualitative study that was conducted in patients with T2D who switched to IDeg from another basal insulin [12]. In this study, four major factors were identified that contributed to patients' sense of "feeling better": a reduced sense of diabetes as burdensome and requiring excessive attention; enhanced feelings of adaptability and freedom; increased sense of security, particularly around concerns related to hypoglycemia; and a greater sense of physical well-being [12]. A review of the literature and of patient blogs [13-17] led to the identification of one additional domain, sleep quality, which appears to influence QoL.

To further explore and validate these observations, we sought to determine in a more quantitative fashion the unique domains related to the experience of "feeling better" when using IDeg, as compared with the patient's previous basal insulin. Five QoL domains were examined: three were diabetes-specific (perceptions of safety with regards to hypoglycemia, sense of diabetes as burdensome, feelings of freedom and flexibility in regard to diabetes management) and two were generic (well-being and sleep quality).

The primary aim of this study was to examine the impact between QoL scores with switching from previous basal insulin use to the use of IDeg, and if this was affected by the nature of the reason for switching, i.e., if it was primarily due to lack of health insurance coverage for the previous insulin. Secondary aims included exploring the predictors of improved QoL after switching to IDeg and the correlation of sleep quality with other patient-reported outcome (PRO) measures.

\section{Methods}

\section{Study design and population}

This study was a single-assessment, cross-sectional, online survey of patients with T2D in the USA who were taking IDeg. Eligible patients were aged $\geq 18$ years, had been taking IDeg for $\geq 3$ months, had previously used at least one other basal insulin before taking IDeg, and had first started taking insulin $\geq 2$ years after being diagnosed with T2D. Patients were identified from a commercially available diabetes patient panel and were invited by email to participate. A target of collecting at least 150 completed surveys was set based on statistical power calculations using 2017 T2D prevalence rates, to permit subgroup analyses, and to ensure a diverse patient sample.

Respondents were asked to provide written informed consent and to complete a short eligibility questionnaire. All respondents who met the eligibility criteria and completed the survey were included in the study analyses. For participation in this study, they received an honorarium of US\$10 in the form of reward points that were redeemable for a gift card or cash. Confidentiality and privacy of the study data were managed in accordance with Health Insurance Portability and Accountability Act of 1996 regulations, and all study analyses used anonymized data stored on secure servers. Ethics approval of the study protocol was obtained from New England Independent Review Board. As this was a noninterventional study, trial registration was not required.

\section{Survey questions}

The survey instrument was developed jointly by IQVIA and Novo Nordisk, Inc. (Plainsboro, NJ, USA); it was administered by Nielsen Holdings, Inc. (New York, NY, USA) from March 29 to May 7, 2018. The survey was developed by first performing a focused literature review to identify generic or diabetes-specific PRO instruments that mapped to the domains of interest in this study. IQVIA and Novo Nordisk, Inc. then collaborated with a diabetes psychologist (WHP) to judge the clarity, relevance, value, and priority of the items selected.

The survey consisted of 70 questions and took approximately 20 min to complete. To identify any potential issues, the content was reviewed by two eligible patients. The survey's structure and scoring scheme are summarized in Online Resource 1. Data on demographics and clinical history were obtained both from the eligibility questionnaire and from 12 of the questions in the main survey itself. The remaining 58 questions in the survey asked patients about the five QoL domains that were the focus of this study. 
To allow comparison with previous studies, the domains of "well-being" and "sense of diabetes as burdensome" were assessed using modified versions of the World Health Organization (Five) Well-Being Index (WHO-5) and Diabetes Distress Scale (DDS), respectively, as versions of these instruments had previously been used to assess these domains in a large online survey of patients with type 1 diabetes (T1D) [18]. The WHO-5 is a commonly used fiveitem generic instrument that has been validated as a measure of well-being in patients with T1D and T2D [19, 20]. A change of 10 points in WHO-5 total percentage score is considered to be clinically relevant $[21,22]$. The DDS comprises four subscales (emotional burden, physicianrelated distress, regimen-related distress, and interpersonal distress) and has been used both as an outcome measure in several clinical studies, and as an instrument to facilitate communication with patients in clinical practice [23]. This instrument is a validated measure of diabetes-related emotional distress for both T1D and T2D populations [24, 25]. Only the emotional burden and regimen-related distress subscales were included in the present study.

"Sense of safety with regards to hypoglycemia" was assessed using a modified version of the Hypoglycemia Attitudes and Behavior Scale (HABS). This instrument consists of three subscales (avoidance, confidence, and anxiety) and has been validated for use in adults with T2D $[26,27]$. In our study, we included only the confidence and anxiety subscales.

The standard versions of the WHO-5, DDS, and HABS all ask patients to rate how they feel about their wellbeing, diabetes-related distress, or hypoglycemia, respectively, during a single specified time period-“over the last 2 weeks" (WHO-5), "during the past month" (DDS), or "current feelings" (HABS) [21, 23, 26]. We modified these three instruments so that patients rated each item twice. First, we asked patients to think back to when they were on their previous insulin (directly before switching to IDeg) and to indicate how they felt at that time. Second, we asked them to indicate how they had been feeling over the last 2 weeks (WHO-5), during the past month (DDS), or at the current time (HABS) while using IDeg.

Our review of the literature did not yield any diabetesspecific PRO measure that could map to "feelings of freedom and flexibility." Therefore, we assessed this domain using newly generated questions that were designed based on patients' feedback in the previous qualitative study that investigated QoL in patients who switched to IDeg from another basal insulin [12]. Patients were asked to indicate the degree to which they agreed or disagreed with the three following statements, thinking back to when they were on their previous basal insulin and while using IDeg: (1) feel pressured to eat snacks to avoid low blood sugar problems; (2) feel restricted about if and/or when I can exercise; (3) feel like I can't be as spontaneous in my life as I want to be.

Each item in the modified WHO-5, HABS, and DDS, and in the "feelings of freedom and flexibility" domain was assessed using either a five- or six-point Likert-type scale (see Online Resource 1). Sleep quality was quantified by asking patients their average number of hours of restful sleep, thinking back to when they were on their previous basal insulin and while using IDeg.

\section{Statistical analysis}

Descriptive statistics were used to characterize the data. Categorical measures were reported using frequency (number of cases) and percentage of the total number of patients observed in each category. Continuous measures were reported using mean, median, standard deviation (SD), and range. Analyses of PRO scores were conducted in the whole study population and in patient subgroups defined according to whether switching to IDeg was due to the previous basal insulin no longer being covered by the patient's insurance company. For comparative analyses (overall and subgroup), statistical significance was determined by the Wilcoxon signed-rank test for continuous variables. A $P$-value $<0.05$ was considered statistically significant. Imputations or substitutions were not performed for missing data.

Both univariate and multivariable linear regression analyses were performed to identify whether any of five key variables (age, sex, ethnicity, T2D duration, and length of time on IDeg) were associated with the difference in scores (defined as score while using the previous basal insulin minus the score while using IDeg) for each of the PRO scales. These five particular variables were significantly associated with the outcomes based on the univariate analysis and served as the independent variables in each multivariable model assessed. In addition, Pearson correlation coefficients were used to assess the correlation between the difference in the average number of hours of restful sleep and the difference in scores on each of the other PRO scales, as we wondered whether improved sleep might have resulted from reductions in hypoglycemia concerns.

\section{Results}

A total of 6378 patients were screened for eligibility, and 152 (2.4\%) met the eligibility criteria and completed the survey. As IDeg was new to the market, a large number of patients needed to be screened to reach the target sample size. The most common reasons for not meeting eligibility criteria were that the patient had started taking insulin $<2$ years after being diagnosed with T2D or that the patient was not currently taking IDeg. The study population ranged in age from 
18 to 86 years, had a mean $(S D)$ age of $48.6(15.7)$ years, and included a similar number of female $(n=78 ; 51.3 \%)$ and male patients $(n=74 ; 48.7 \%$; Table 1$)$. The majority of the sample $(55.3 \%)$ reported a T2D duration $\geq 6$ years. The most commonly used previous basal insulins were insulin glargine U-100 and insulin NPH (Table 1). Approximately two-thirds of patients had been using IDeg for 7 months or longer.

\section{Impact on QoL after switching to IDeg}

Patients reported statistically significantly higher scores on all five WHO-5 items and on the WHO-5 total raw score while using IDeg compared with their previous basal insulin (all $P<0.0001$; Fig. 1). The mean improvement in WHO-5 total raw score was 3.0 points, equivalent to an improvement of 12.0 points in WHO-5 total percentage score. On the HABS confidence subscale, all item scores, as well as the mean subscale score, were also significantly higher while using IDeg than with the previous basal insulin (all $P<0.0001$; Fig. 2a). On the HABS anxiety subscale (Fig. 2b), DDS emotional burden subscale (Fig. 3a), and DDS regimen-related distress subscale (Fig. 3b), all item scores as well as the mean subscale score were significantly lower while using IDeg than with the previous basal insulin (all $P<0.0001$ ). Improved QoL after switching to IDeg was reported by $\geq 50 \%$ of patients on the WHO-5, HABS confidence and anxiety subscales, and DDS emotional burden and regimen-related distress subscales (Online Resource 2).

Scores on all three items assessing patients' feelings of freedom and flexibility were significantly higher while using IDeg compared with the previous basal insulin (all $P<0.0001$; Fig. 4). Patients also reported a significantly greater average number of hours of restful sleep while using IDeg compared with their previous basal insulin [mean 6.6 $(S D 2.0)$ vs. 5.5 ( $S D 1.8) ; P<0.0001]$. An increased number of hours of restful sleep after switching to IDeg was reported by the majority of patients (59.2\%; Online Resource 2 ).

Patients who were involuntarily switched to IDeg (i.e., previous basal insulin was no longer covered by insurance; $N=43$ ) reported significant improvements in HABS anxiety subscale score $(P=0.043)$, DDS emotional burden subscale score $(P=0.023)$, feelings of freedom and flexibility total score $(P=0.0039)$, and average number of hours of restful sleep $(P=0.0002$; Table 2). Patients who did not switch due to insurance changes $(N=108)$ reported significant improvements in all QoL domains (Table 2; all $P<0.0001$ ).

\section{Predictors of improved QoL after switching to IDeg}

The univariate regression analyses found that a T2D duration of $<3$ years, non-white ethnicity, and younger age were statistically significant predictors of a greater level of improvement in QoL in multiple domains after switching to IDeg.
In the multivariable regression analyses, a T2D duration of $<3$ years and non-white ethnicity remained statistically significant predictors of a greater level of improvement in QoL; however, these varied by QoL domain.

\section{Correlation of sleep quality with other PRO scales}

A greater amount of restful sleep was found to be significantly correlated with improved QoL as assessed by WHO-5 total raw score, HABS and DDS mean subscale scores, and each item in the feelings of freedom and flexibility domain (Table 3).

\section{Discussion}

Guidelines from the American Association of Clinical Endocrinologists/American College of Endocrinology [2] and American Diabetes Association [28] stress that the choice of insulin therapy should be a collaborative decision between the healthcare provider and the patient. Therefore, given the importance of the patient's voice, we conducted the present study to quantitatively assess change in QoL across a broad range of domains that previous research highlighted as being of importance to patients. In particular, anecdotal evidence has indicated that patients with T2D often report "feeling better" when treated with IDeg. We therefore sought to understand what this interesting finding might refer to and how we might assess this more carefully. Of the previous studies that quantitatively investigated how basal insulin affects QoL in patients with T2D [11, 29, 30], most have used relatively broad, generic measures. One strength of our study is that we included a range of diabetes-specific PRO instruments to assess QoL, thus providing additional information on the specific issues that patients with T2D face, such as concerns related to hypoglycemia.

In the previous qualitative QoL study, patients who had switched to IDeg from another basal insulin reported greater energy and less fatigue, a heightened sense of security regarding hypoglycemia-related concerns, a reduced sense of diabetes as being burdensome, and enhanced feelings of adaptability and freedom [12]. The results in the current study support these findings. Patients who switched to IDeg from a previous basal insulin appeared to experience significantly improved well-being and feelings of freedom and flexibility. Specifically, patients reported they felt less pressured to eat snacks to avoid low blood glucose problems, felt less restricted about exercising, and felt they could be more spontaneous in their lives. Patients also reported significantly increased confidence and less anxiety around potential issues relating to hypoglycemia, such as safety when away from home or while driving. Patients also felt their diabetes became significantly less burdensome and 
Table 1 Patient demographics and clinical characteristics

\begin{tabular}{|c|c|}
\hline Characteristic & $\begin{array}{l}\text { Total study } \\
\text { population } \\
(N=152)\end{array}$ \\
\hline \multicolumn{2}{|l|}{ Age, years } \\
\hline Mean \pm SD & $48.6 \pm 15.7$ \\
\hline Median (range) & $46.0(18-86)$ \\
\hline \multicolumn{2}{|l|}{ Age group, $n(\%)$} \\
\hline $18-39$ years & $55(36.2)$ \\
\hline $40-64$ years & $65(42.8)$ \\
\hline$\geq 65$ years & $32(21.1)$ \\
\hline \multicolumn{2}{|l|}{ Sex, $n(\%)$} \\
\hline Female & $78(51.3)$ \\
\hline Male & $74(48.7)$ \\
\hline \multicolumn{2}{|l|}{ Ethnicity, $n(\%)^{\mathrm{a}}$} \\
\hline White & $110(72.4)$ \\
\hline Hispanic, Latino, or Spanish origin & $19(12.5)$ \\
\hline Black or African American & $19(12.5)$ \\
\hline Asian & $6(3.9)$ \\
\hline American Indian or Alaskan Native & $3(2.0)$ \\
\hline Middle Eastern or North African & $2(1.3)$ \\
\hline Native Hawaiian or other Pacific Islander & $1(0.7)$ \\
\hline \multicolumn{2}{|l|}{ US region, $n(\%)$} \\
\hline South & $69(45.4)$ \\
\hline Midwest & $35(23.0)$ \\
\hline Northeast & $25(16.4)$ \\
\hline West & $23(15.1)$ \\
\hline \multicolumn{2}{|l|}{ Employment status, $n(\%)$} \\
\hline Employed full-time & $79(52.0)$ \\
\hline Retired & $43(28.3)$ \\
\hline Employed part-time & $12(7.9)$ \\
\hline Homemaker & $12(7.9)$ \\
\hline Other & $6(3.9)$ \\
\hline \multicolumn{2}{|l|}{ Highest degree of education, $n(\%)$} \\
\hline Less than high school diploma & $2(1.3)$ \\
\hline High school graduate (diploma or GED) & $31(20.4)$ \\
\hline Some college or associate degree & $43(28.3)$ \\
\hline Bachelor's degree & $46(30.3)$ \\
\hline Some graduate school & $7(4.6)$ \\
\hline Master's degree or higher & $23(15.1)$ \\
\hline \multicolumn{2}{|l|}{ Type of health insurance, $n(\%)^{\mathrm{a}}$} \\
\hline Insurance coverage through a current or former employer & $71(46.7)$ \\
\hline Medicare & $41(27.0)$ \\
\hline Individual/family insurance plans/Healthcare.gov/Affordable Care Act (i.e., coverage purchased directly by patient) & $23(15.1)$ \\
\hline Insurance coverage through spouse's employer & $16(10.5)$ \\
\hline Medicaid (MediCal for California residents) & $14(9.2)$ \\
\hline Other & $10(6.6)$ \\
\hline Not sure & $2(1.3)$ \\
\hline \multicolumn{2}{|l|}{ Current marital/relationship status, $n(\%)$} \\
\hline Married & $94(61.8)$ \\
\hline Single, never married & $36(23.7)$ \\
\hline Widowed & $13(8.6)$ \\
\hline
\end{tabular}


Table 1 (continued)

Characteristic

Total study population

$(N=152)$

Separated

$5(3.3)$

Living with partner

4 (2.6)

T2D duration, $n(\%)$

$<3$ years

$11(7.2)$

$3-5$ years

57 (37.5)

6-10 years

$39(25.7)$

$11-15$ years

$22(14.5)$

$\geq 16$ years

$23(15.1)$

Medical conditions diagnosed prior to using a basal insulin (self-reported), $n(\%)^{\mathrm{a}}$

High blood pressure

$81(53.3)$

High cholesterol or triglycerides

77 (50.7)

Obesity

55 (36.2)

Depression

48 (31.6)

Anxiety

43 (28.3)

None of the above

27 (17.8)

Prior basal insulin, $n(\%)^{\mathrm{a}}$

Insulin glargine U-100 (Lantus $\left.{ }^{\circledR}\right)$

$64(42.1)$

Insulin NPH

$50(32.9)$

Insulin detemir

42 (27.6)

Insulin glargine U-300

$35(23.0)$

Other

7 (4.6)

Switch to IDeg because previous basal insulin no longer covered by patient's insurance company, $n$ (\%)

Yes

$43(28.3)$

No

$108(71.1)$

Don't know

$1(0.7)$

IDeg duration, $n(\%)$

3-6 months

$50(32.9)$

7-12 months

48 (31.6)

$>12$ months

$54(35.5)$

IDeg concentration, $n(\%)$

U-100

$82(53.9)$

U-200

68 (44.7)

Don't know

$2(1.3)$

Units of IDeg taken each day ${ }^{\mathrm{b}}$

Mean \pm SD

$33.1 \pm 41.7$

Median (range)

$8.5(1-160)$

Last HbA1c level, $n(\%)$

$\leq 5.0 \%$

$0(0)$

$5.1-6.0 \%$

7 (4.6)

$6.1-7.0 \%$

$41(27.0)$

$7.1-8.0 \%$

45 (29.6)

$8.1-9.0 \%$

21 (13.8)

9.1-10.0\%

$15(9.9)$

$10.1-11.0 \%$

7 (4.6)

$11.1-12.0 \%$

6 (3.9)

$\geq 12.1 \%$

$0(0)$

Don't know

$10(6.6)$ 
Table 1 (continued)

Characteristic

Blood sugar test frequency (over last month), $n(\%)$

Less than every day

Daily

Twice a day

Three times a day

More than three times a day $8(5.3)$

GED general education development, HbAlc glycated hemoglobin, IDeg insulin degludec, $N P H$ neutral protamine Hagedorn, $S D$ standard deviation, $T 2 D$ type 2 diabetes

${ }^{a}$ Patients could select more than one option, so the percentages sum to more than $100 \%$

${ }^{\mathrm{b}} N=142$

Fig. 1 WHO-5 item and total raw scores $(N=152)$. Higher scores indicate better QoL.

Total raw score is the sum of the 5-item scores. $* P<0.0001$ for score while using IDeg vs. score while using previous basal insulin. IDeg insulin degludec, $Q o L$ quality of life, $S D$ standard deviation, WHO-5 World Health Organization (Five) Well-Being Index

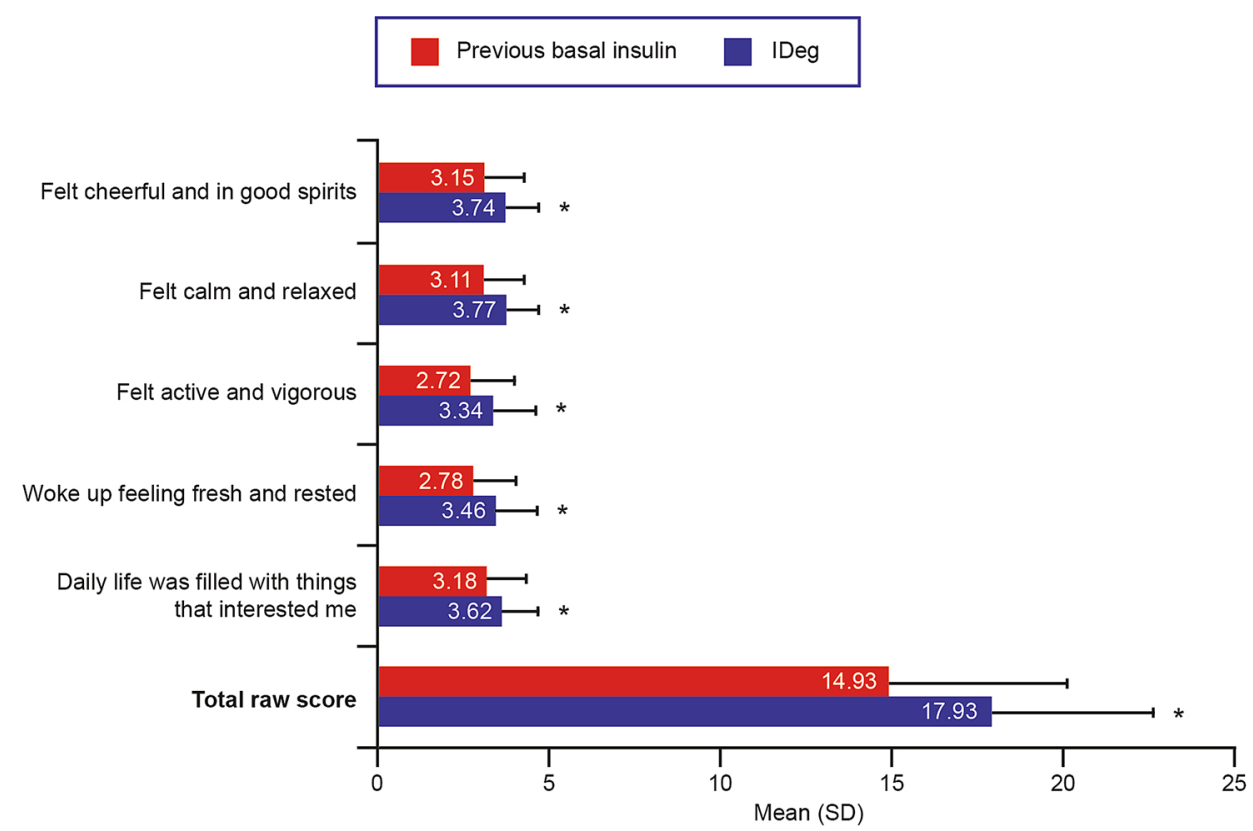

experienced significantly less distress related to the day-today management of their diabetes.

In addition, sleep quality (hours of restful sleep) was also improved. Of note, sleep quality was associated with improved QoL on all of the other PRO domains assessed. Since factors such as improved glycemic control or reduced nocturnal glycemic variability could result in both improved sleep quality and in improved QoL, further studies to investigate these associations are warranted. One interesting area of future research would be to investigate the influence of basal insulin use on QoL, especially hypoglycemia outcomes and sleep quality, with more objective measures (e.g., actigraphy, polysomnography and continuous glucose monitoring).

Patients may have switched to IDeg due to dissatisfaction (their own and/or their healthcare providers') with their original insulin product. In that case, the observed QoL improvements may represent merely a regression to the mean, and therefore not resulting from any benefits accruing from the use of IDeg. To explore this issue, we conducted a set of secondary analyses to examine QoL improvement in that subset of patients (28.3\% of the study sample) who were forced to change to IDeg due to insurance changes. We found that significant QoL changes were reported in this subset of patients, as well as in that subset who chose to switch, supporting a positive association between QoL and the use of IDeg.

Predictors of QoL improvements after switching to IDeg warrant further exploration. While it is understandable that a short experience with diabetes may be related to a more hopeful disposition on QoL in general, it is unclear whether cultural factors related to non-white ethnicity, for example, may contribute to improved QoL as well. We suspect that 
Fig. 2 HABS item and mean subscale scores $(N=152)$. a On the confidence subscale, higher scores indicate better QoL (i.e., greater confidence). $\mathbf{b}$ On the anxiety subscale, lower scores indicate better quality of life (i.e., less anxiety). $* P<0.0001$ for score while using IDeg vs. score while using previous basal insulin. HABS Hypoglycemia Attitudes and Behavior Scale, IDeg insulin degludec, $Q o L$ quality of life, $S D$ standard deviation
Previous basal insulin IDeg

(a)

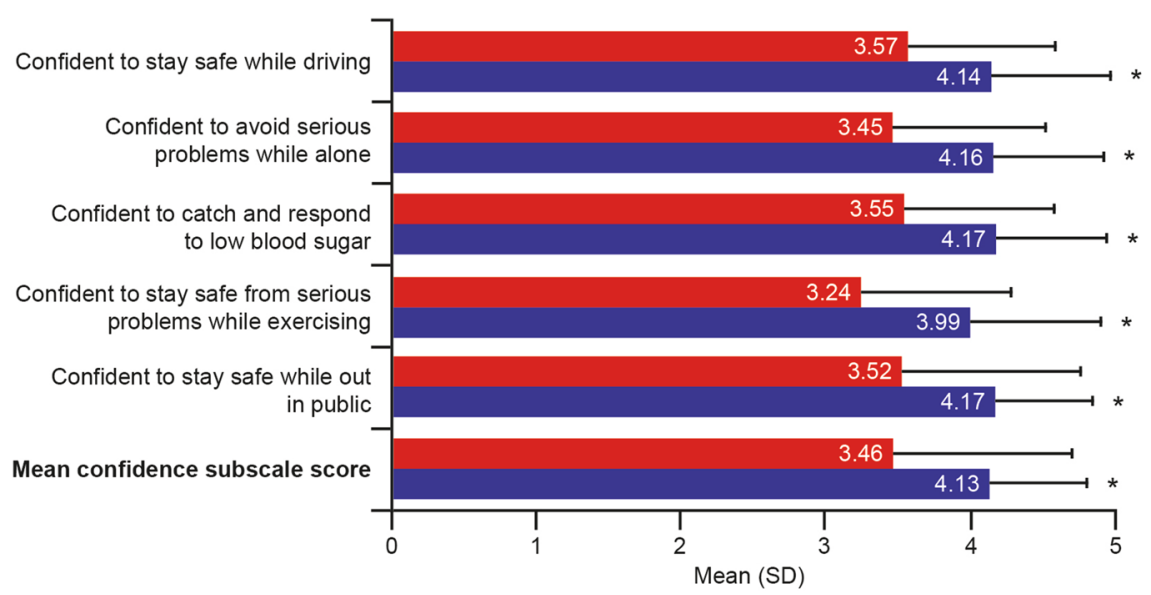

(b)

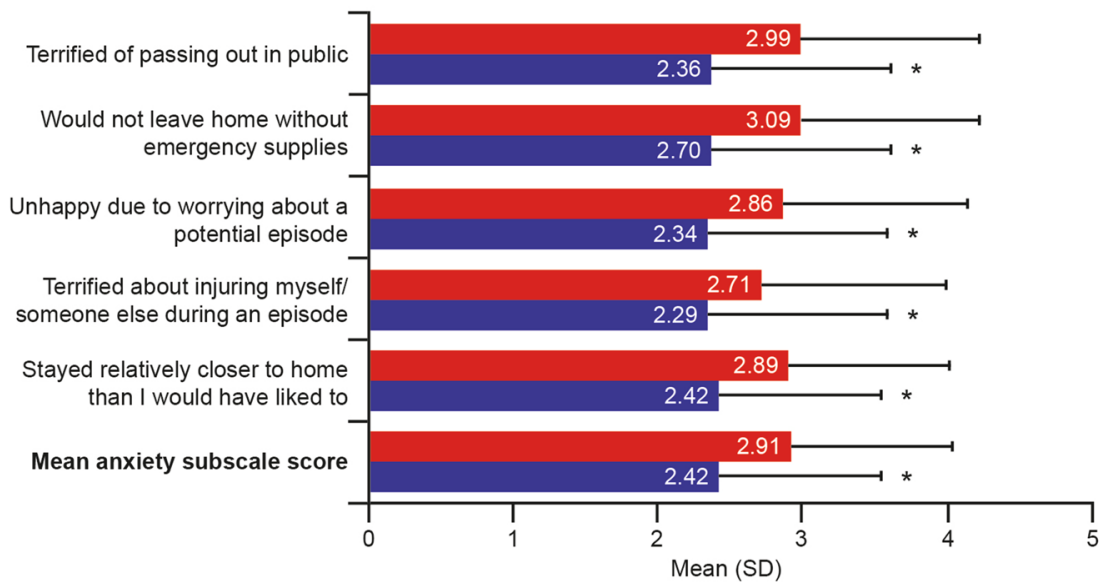

IDeg-associated QoL improvement may contribute to greater insulin adherence and persistence over time. Though this could not be evaluated in the current study, we hope to do so in future studies.

\section{Limitations}

Only $2.4 \%$ of patients who were screened for eligibility met the inclusion criteria and completed the online survey. Further, the participants were selected from a convenience sample via a commercially available diabetes patient panel; therefore, their results may not be generalizable to the broader IDeg-using population due to potentially different demographic characteristics including a higher educational level and income, which could impact QoL. In addition, all data were self-reported by patients, including the diagnosis of T2D. However, the eligibility questionnaire asked multiple questions about the patient's history and treatment of T2D, which increases our confidence that our study recruited an appropriate study population.
Other clinical information such as previous basal insulin usage, T2D duration, and length of time using IDeg were also all self-reported. Other health conditions were not assessed in the survey, thus QoL could be confounded by the presence of comorbidities. Furthermore, the study was cross-sectional in nature; patients were not surveyed before and after starting IDeg but were asked to recall their perceptions thinking back to when they had been using their previous basal insulin, which likely introduced recall bias. Previous studies have found that when patients with insulin-treated T2D are asked to self-report the incidence of hypoglycemic events retrospectively, they underreport the incidence compared with when they are asked to selfreport prospectively $[31,32]$. Recall bias may be due to a number of factors, including the length of recall period [33]. In the present study, the fact that the majority of patients $(64.5 \%)$ had switched to IDeg within a year, and nearly a third $(32.9 \%)$ had switched to IDeg only within the last 3-6 months, may mitigate the length of the recall period as a source of recall bias. 
Fig. 3 DDS item and mean subscale scores $(N=152)$. On both the emotional burden subscale (a) and the regimen-related distress subscale (b), lower scores indicate better QoL (i.e., less burden and lower distress). * $P<0.0001$ for score while using IDeg vs. score while using previous basal insulin. $D D S$ Diabetes Distress Scale, IDeg insulin degludec, $Q o L$ quality of life, $S D$ standard deviation
Fig. 4 Feelings of freedom and flexibility: item and total scores $(N=152)$. Higher scores indicate better QoL. * $P<0.0001$ for score while using IDeg vs. score while using previous basal insulin. IDeg insulin degludec, $Q o L$ quality of life, $S D$ standard deviation
Previous basal insulin

IDeg

(a)

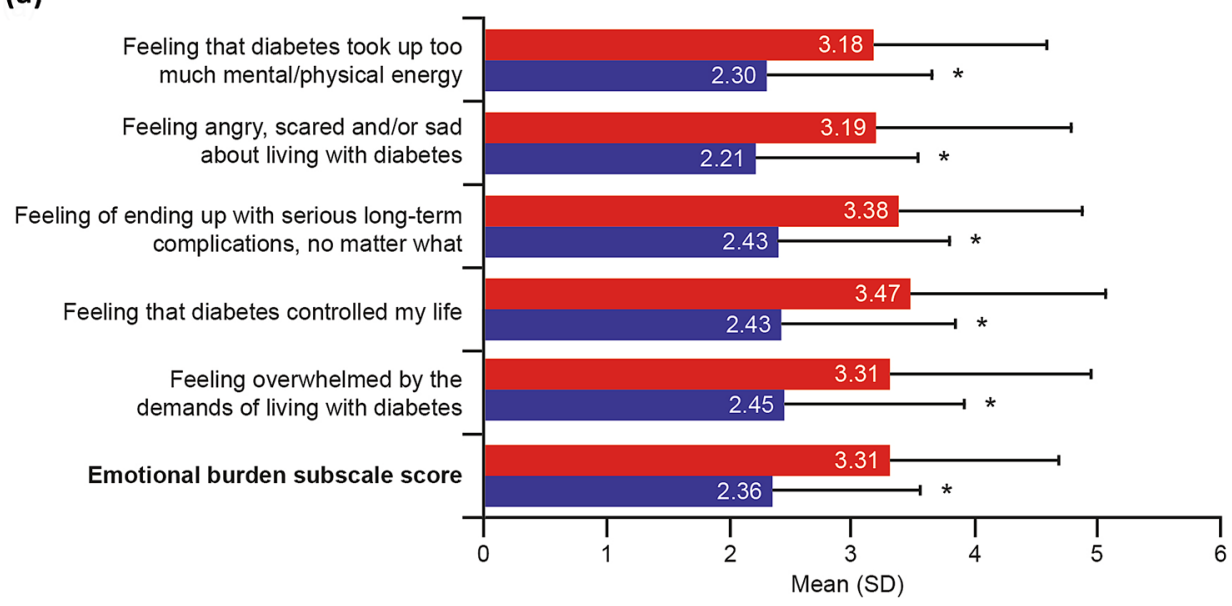

(b)
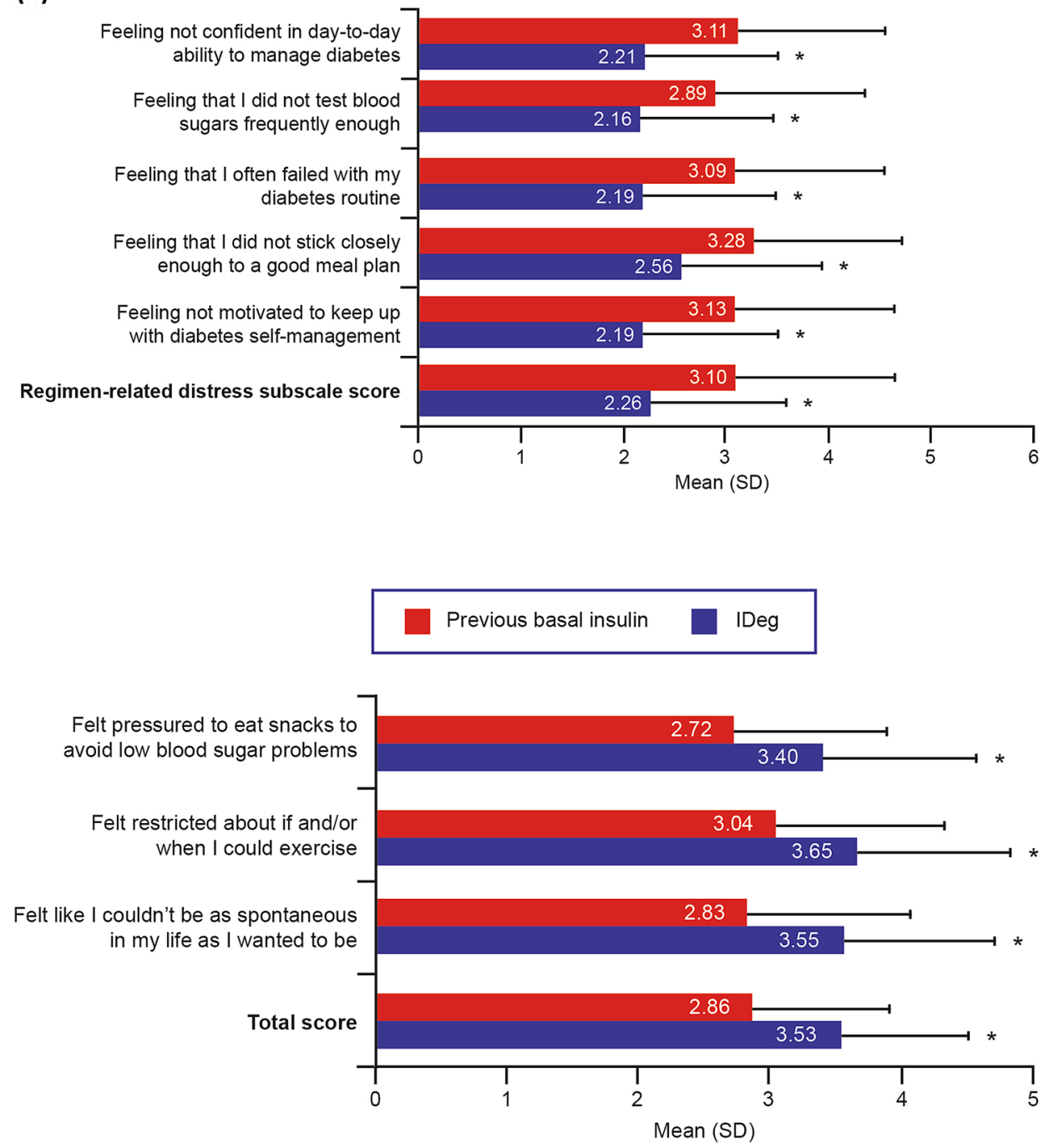
Table 2 PRO scores in patients based on insurance-determined basal insulin coverage

\begin{tabular}{|c|c|c|c|c|c|c|}
\hline \multirow[t]{3}{*}{ PRO score, mean $\pm S D$} & \multicolumn{6}{|c|}{$\begin{array}{l}\text { Switched to IDeg because previous basal insulin was no longer covered by the patient's insur- } \\
\text { ance company }\end{array}$} \\
\hline & \multicolumn{3}{|l|}{ Yes $(N=43)$} & \multicolumn{3}{|l|}{ No $(N=108)$} \\
\hline & Previous basal insulin & IDeg & $P$-value ${ }^{\mathrm{a}}$ & Previous basal insulin & IDeg & $P$-value ${ }^{\mathrm{a}}$ \\
\hline WHO-5 total raw score & $17.00 \pm 4.24$ & $17.35 \pm 4.31$ & 0.4887 & $14.10 \pm 5.28$ & $18.19 \pm 4.83$ & $<0.0001$ \\
\hline HABS mean confidence subscale score & $3.71 \pm 0.72$ & $3.89 \pm 0.66$ & 0.1202 & $3.37 \pm 0.96$ & $4.22 \pm 0.65$ & $<0.0001$ \\
\hline HABS mean anxiety subscale score & $2.96 \pm 1.11$ & $2.80 \pm 1.00$ & 0.043 & $2.89 \pm 1.14$ & $2.26 \pm 1.13$ & $<0.0001$ \\
\hline DDS mean emotional burden subscale score & $3.24 \pm 1.16$ & $2.92 \pm 1.17$ & 0.023 & $3.34 \pm 1.47$ & $2.14 \pm 1.17$ & $<0.0001$ \\
\hline $\begin{array}{l}\text { DDS mean regimen-related distress subscale } \\
\text { score }\end{array}$ & $3.05 \pm 1.09$ & $2.74 \pm 1.18$ & 0.3328 & $3.12 \pm 1.29$ & $2.08 \pm 1.09$ & $<0.0001$ \\
\hline Feelings of freedom and flexibility total score & $2.78 \pm 0.98$ & $3.25 \pm 0.86$ & 0.0039 & $2.90 \pm 1.07$ & $3.65 \pm 1.02$ & $<0.0001$ \\
\hline Average number of hours of restful sleep & $5.40 \pm 2.12$ & $6.37 \pm 2.43$ & 0.0002 & $5.55 \pm 1.71$ & $6.69 \pm 1.73$ & $<0.0001$ \\
\hline
\end{tabular}

DDS Diabetes Distress Scale; HABS Hypoglycemia Attitudes and Behavior Scale; IDeg insulin degludec; PRO patient-reported outcome; SD standard deviation; WHO-5 World Health Organization (Five) Well-Being Index

${ }^{\text {a }} P$-value for PRO score while using IDeg vs. score while using the previous basal insulin

Table 3 Correlation between sleep quality and PRO scales

\begin{tabular}{lcr}
\hline & $\begin{array}{l}\text { Correlation } \\
\text { coefficient }^{\mathrm{a}}\end{array}$ & $P$-value \\
\hline Sense of physical well-being (WHO-5) & $0.40^{\mathrm{b}}$ & $<0.0001$ \\
Sense of safety (HABS) & $0.54^{\mathrm{b}}$ & $<0.0001$ \\
Confidence subscale & $-0.32^{\mathrm{c}}$ & $<0.0001$ \\
$\quad$ Anxiety subscale & & $<0.0001$ \\
Sense of diabetes as burdensome (DDS) & $-0.55^{\mathrm{c}}$ & $<0.0001$ \\
Emotional burden subscale & $-0.53^{\mathrm{c}}$ & \\
Regimen-related distress subscale & & 0.0004 \\
Feelings of freedom and flexibility & $0.28^{\mathrm{b}}$ & 0.0002 \\
Felt pressured to eat snacks to avoid low blood sugar problems & $0.29^{\mathrm{b}}$ & $<0.0001$ \\
Felt restricted about if and/or when I could exercise & $0.37^{\mathrm{b}}$ & \\
Felt like I couldn't be as spontaneous in my life as I wanted to be & & \\
\hline
\end{tabular}

DDS Diabetes Distress Scale, HABS Hypoglycemia Attitudes and Behavior Scale, IDeg insulin degludec, $P R O$ patient-reported outcomes, $W H O-5$ World Health Organization (five) Well-Being Index

${ }^{a}$ Correlation coefficients show the correlation between the difference in the average number of hours of restful sleep (i.e., number of hours while using the previous basal insulin minus number of hours while using IDeg) and the difference in PRO scores (i.e., score while using the previous basal insulin minus score while using IDeg)

b On the WHO-5 and HABS confidence subscales, and "feelings of freedom and flexibility" scale, a positive correlation coefficient indicates that a greater amount of restful sleep correlates with better quality of life

${ }^{c}$ On the HABS anxiety subscale and both DDS subscales, a negative correlation coefficient indicates that a greater amount of restful sleep correlates with better quality of life (i.e., less anxiety/distress)

\section{Conclusions}

In this quantitative study, patients with $\mathrm{T} 2 \mathrm{D}$ reported statistically significantly better QoL while using IDeg than while using their previous basal insulin across all the domains assessed. Study limitations include possible recall bias in cross-sectional, patient-reported data. The findings of our study highlight the specific domains and instruments that should be used in future prospective outcome studies to uncover positive responses that may have been missing in past studies. Significant selfreported improved scores after switching to IDeg included enhanced feelings of well-being, more confidence and less anxiety regarding potential issues with hypoglycemia, less diabetes burdensomeness, less regimen-related distress, greater feelings of freedom and flexibility in their daily lives, and more hours of restful sleep. 
Acknowledgements Elizabeth Powers of IQVIA (New York, NY, USA) contributed to the study design and survey development. Writing assistance was provided by Diane Kwiatkoski and Reza Sayeed of IQVIA (Parsippany, NJ, USA) and funded by Novo Nordisk Inc.

Author contributions $\mathrm{CU}, \mathrm{MR}, \mathrm{CMH}, \mathrm{SK}$, and WHP participated in study design, data analysis and interpretation, and manuscript preparation. MD participated in study design, data interpretation, and manuscript preparation. $\mathrm{CH}$ participated in study design, survey development, data interpretation, and manuscript preparation. All named authors meet the International Committee of Medical Journal Editors criteria for authorship for this article, take responsibility for the integrity of the work as a whole, and have given their approval for this version to be published.

Funding The funding was supported by Novo Nordisk Inc.

Data availability The datasets analyzed during the current study are available from the corresponding author on reasonable request.

\section{Compliance with ethical standards}

Conflict of interest Chioma Uzoigwe, Michael Radin, and Carol M. Hamersky are employed by Novo Nordisk, Inc., the study sponsor. Mitch DeKoven and Swapna Karkare are employed by IQVIA, which received funding from Novo Nordisk, Inc. for work performed on this study under a contract between IQVIA and Novo Nordisk, Inc. Cassie Holt was employed by IQVIA when the study was conducted; she is now employed by Novo Nordisk, Inc. William H. Polonsky served as a consultant and has received consultant fees from Novo Nordisk. William H. Polonsky has also served as a consultant for Sanofi, and Eli Lilly and Company.

Ethical approval Central Institutional Review Board approval was obtained from the New England Independent Review Board on October 30, 2017. The study conformed with the Helsinki Declaration of 1964, as revised in 2013, concerning human and animal rights, and Springer's policy concerning informed consent has been followed.

Informed consent Informed written consent was obtained from all individual participants included in the study.

Open Access This article is licensed under a Creative Commons Attribution 4.0 International License, which permits use, sharing, adaptation, distribution and reproduction in any medium or format, as long as you give appropriate credit to the original author(s) and the source, provide a link to the Creative Commons licence, and indicate if changes were made. The images or other third party material in this article are included in the article's Creative Commons licence, unless indicated otherwise in a credit line to the material. If material is not included in the article's Creative Commons licence and your intended use is not permitted by statutory regulation or exceeds the permitted use, you will need to obtain permission directly from the copyright holder. To view a copy of this licence, visit http://creativecommons.org/licenses/by/4.0/.

\section{References}

1. American Diabetes Association. (2020). 9. Pharmacologic approaches to glycemic treatment: Standards of medical care in diabetes: 2020. Diabetes Care, 43(Suppl 1), S98-S110.

2. Garber, A. J., Abrahamson, M. J., Barzilay, J. I., Blonde, L., Bloomgarden, Z. T., Bush, M. A., et al. (2020). Consensus statement by the American Association of Clinical Endocrinologists and American College of Endocrinology on the comprehensive type 2 diabetes management algorithm: 2020 executive summary. Endocrine Practice, 26, 107-139.

3. Novo Nordisk Inc. (2019). Tresiba ${ }^{\circledR}$ (insulin degludec injection) $100 \mathrm{U} / \mathrm{mL}, 200 \mathrm{U} / \mathrm{mL}$. US prescribing information. Plainsboro, NJ: Novo Nordisk Inc.

4. Mauricio, D., \& Hramiak, I. (2018). Second-generation insulin analogues: A review of recent real-world data and forthcoming head-to-head comparisons. European Journal of Endocrinology, 14, 2-9.

5. Haahr, H., \& Heise, T. (2014). A review of the pharmacological properties of insulin degludec and their clinical relevance. Clinical Pharmacokinetics, 53, 787-800.

6. Heise, T., Nørskov, M., Nosek, L., Kaplan, K., Famulla, S., \& Haahr, H. L. (2017). Insulin degludec: lower day-to-day and within-day variability in pharmacodynamic response compared with insulin glargine $300 \mathrm{U} / \mathrm{mL}$ in type 1 diabetes. Diabetes, Obesity and Metabolism, 19, 1032-1039.

7. Zhang, X. W., Zhang, X. L., Xu, B., \& Kang, L. N. (2018). Comparative safety and efficacy of insulin degludec with insulin glargine in type 2 and type 1 diabetes: A meta-analysis of randomized controlled trials. Acta Diabetologica, 55, 429-441.

8. Heller, S., Mathieu, C., Kapur, R., Wolden, M. L., \& Zinman, B. (2016). A meta-analysis of rate ratios for nocturnal confirmed hypoglycaemia with insulin degludec vs. insulin glargine using different definitions for hypoglycaemia. Diabetes Medicine, 33, 478-487.

9. Marso, S. P., McGuire, D. K., Zinman, B., Poulter, N. R., Emerson, S. S., Pieber, T. R., et al. (2017). Efficacy and safety of degludec versus glargine in type 2 diabetes. New England Journal of Medicine, 377, 723-732.

10. Cornell, S. (2009). Exploring the quality-of-life benefits with insulin analog use. Internet Journal of Family Practice, 9, 1.

11. Freemantle, N., Meneghini, L., Christensen, T., Wolden, M. L., Jendle, J., \& Ratner, R. (2013). Insulin degludec improves health-related quality of life (SF-36®) compared with insulin glargine in people with type 2 diabetes starting on basal insulin: A meta-analysis of phase 3a trials. Diabetic Medicine, 30, 226-232.

12. Weatherall, J., Polonsky, W. H., Lanar, S., Knoble, N., HåkanBloch, J., Constam, E., et al. (2018). When insulin degludec enhances quality of life in patients with type 2 diabetes: A qualitative investigation. Health and Quality of Life Outcomes, 16, 87.

13. The Type 2 Experience. (2019). Boldly redefining type 2 diabetes. Retrieved from https://www.facebook.com/TheType2Experience. Accessed 15 Apr 2019.

14. Diabetes Sisters. (2019). Type 2 diabetes. Retrieved from https:// diabetessisters.org/blogs/type-2-diabetes. Accessed 16 Apr 2019.

15. Sarah, K. (2019). Tresiba insulin: I truly feel in control of my diabetes! Retrieved from https://www.diabetesdaily.com/blog/ tresiba-insulin-i-truly-feel-in-control-of-my-diabetes-275825. Accessed 15 Apr 2019.

16. Johnson, S. T., Thiel, D., Al Sayah, F., Mundt, C., Qiu, W., Buman, M. P., et al. (2017). Objectively measured sleep and health-related quality of life in older adults with type 2 diabetes: A cross-sectional study from the Alberta's Caring for Diabetes Study. Sleep Health, 3, 102-106.

17. Luyster, F. S., \& Dunbar-Jacob, J. (2011). Sleep quality and quality of life in adults with type 2 diabetes. Diabetes Education, 37, 347-355.

18. Polonsky, W. H., Hessler, D., Layne, J. E., \& Zisser, H. (2016). Impact of the Omnipod ${ }^{\circledR}$ insulin management system on quality of life: A survey of current users. Diabetes Technology and Therapeutics, 18, 664-670. 
19. Hajos, T. R., Pouwer, F., Skovlund, S. E., Den Oudsten, B. L., Geelhoed-Duijvestijn, P. H., Tack, C. J., et al. (2013). Psychometric and screening properties of the WHO-5 well-being index in adult outpatients with type 1 or type 2 diabetes mellitus. Diabetic Medicine, 30, e63-e69.

20. Hochberg, G., Pucheu, S., Kleinebreil, L., Halimi, S., \& Fructuoso-Voisin, C. (2012). WHO-5, a tool focusing on psychological needs in patients with diabetes: The French contribution to the DAWN study. Diabetes and Metabolism, 38, 515-522.

21. Psychiatric Research Unit, WHO Collaborating Center for Mental Health. (1998). WHO (Five) Well-Being Index (1998 version). Hillerød: Psychiatric Research Unit, WHO Collaborating Center for Mental Health. https://www.psykiatri-regionh.dk/who-5/ Documents/WHO5_English.pdf. Accessed 16 Apr 2019

22. Topp, C. W., Østergaard, S. D., Søndergaard, S., \& Bech, P. (2015). The WHO-5 Well-Being Index: A systematic review of the literature. Psychotherapy and Psychosomatics, 84, 167-176.

23. Behavioral Diabetes Institute. (2019). Distress scale (DDS). Retrieved from https://behavioraldiabetes.org/scales-and-measu res/\#1448434304099-9078f27c-4106. Accessed 16 Apr 2019.

24. Polonsky, W. H., Fisher, L., Earles, J., Dudl, R. J., Lees, J., Mullan, J., et al. (2005). Assessing psychosocial distress in diabetes: Development of the diabetes distress scale. Diabetes Care, 28, 626-631.

25. Schmitt, A., Reimer, A., Kulzer, B., Haak, T., Ehrmann, D., \& Hermanns, N. (2016). How to assess diabetes distress: Comparison of the Problem Areas in Diabetes Scale (PAID) and the Diabetes Distress Scale (DDS). Diabetic Medicine, 33, 835-843.

26. Behavioral Diabetes Institute. (2019). Hypoglycemia attitudes and behavior scale (HABS). Retrieved from https://behavioraldiabe tes.org/scales-and-measures/\#1448435050704-6e22e4b0-81ec. Accessed 16 Apr 2019.

27. Polonsky, W. H., Fisher, L., Hessler, D., \& Edelman, S. V. (2015). Identifying the worries and concerns about hypoglycemia in adults with type 2 diabetes. Journal of Diabetes and Its Complications, 29, 1171-1176.
28. American Diabetes Association. (2020). 1. Improving care and promoting health in populations: Standards of medical care in diabetes: 2020. Diabetes Care, 43(Suppl 1), S7-S13.

29. Hajos, T. R., Pouwer, F., de Grooth, R., Holleman, F., Twisk, J. W., Diamant, M., et al. (2011). Initiation of insulin glargine in patients with type 2 diabetes in suboptimal glycaemic control positively impacts health-related quality of life. A prospective cohort study in primary care. Diabetic Medicine, 28, 1096-1102.

30. Freemantle, N., Evans, M., Christensen, T., Wolden, M. L., \& Bjorner, J. B. (2013). A comparison of health-related quality of life (health utility) between insulin degludec and insulin glargine: A meta-analysis of phase 3 trials. Diabetes, Obesity and Metabolism, 15, 564-571.

31. Kern, W., Holstein, A., Moenninghoff, C., Kienhöfer, J., Riedl, M., \& Kulzer, B. (2017). Self-reported hypoglycaemic events in 2430 patients with insulin-treated diabetes in the German subpopulation of the HAT study. Experimental and Clinical Endocrinology and Diabetes, 125, 592-597.

32. Rudijanto, A., Saraswati, M. R., Yunir, E., Kumala, P., Puteri, H. H., \& Mandang, V. V. (2018). Indonesia cohort of IO HAT study to evaluate diabetes management, control, and complications in retrospective and prospective periods among insulin-treated patients with type 1 and type 2 diabetes. Acta Medica Indonesiana, 50, 26-37.

33. Althubaiti, A. (2016). Information bias in health research: Definition, pitfalls, and adjustment methods. Journal of Multidisciplinary Healthcare, 9, 211-217.

Publisher's Note Springer Nature remains neutral with regard to jurisdictional claims in published maps and institutional affiliations. 